\title{
A CLASS OF NONLINEAR PARTIAL DIFFERENTIAL EQUATIONS AND THEIR PROPERTIES
}

\author{
STEFAN BERGMAN
}

1. The class $\mathcal{C}$ of complex solutions of a linear partial differential equation. Any two real harmonic functions $U(x, y), V(x, y)$-that is, solutions of the Laplace equation - can be combined to form a complex harmonic function $U+i V$. The class of all complex harmonic functions is of no interest, because in effect it possesses no special properties not already possessed by real harmonic functions. However, the theory of analytic functions of a complex variable, which is the subclass of complex harmonic functions where $U$ and $V$ satisfy the Cauchy-Riemann equations, has proved to be a powerful means for the study of real harmonic functions.

There is an analogous situation in the case of real solutions of the general linear equation of elliptic type,

$$
\begin{gathered}
U_{x x}+U_{y y}+A(x, y) U_{x}+B(x, y) U_{y}+C(x, y) U=0, \\
U_{x}=\partial U / \partial x, \cdots .
\end{gathered}
$$

As in the case of the Laplace equation, any two real solutions of this equation can be combined to form a complex solution, and it has been shown $([1 \mathrm{a}, 1 \mathrm{~b}, 1 \mathrm{c}])^{1}$ that there exists a certain subclass $\mathcal{C}$ of complex solutions which frequently aids in the study of real solutions in a manner which bears close analogies to the relationships between analytic functions and real harmonic functions in the case of the Laplace equation. Many properties of functions of class $\mathcal{C}$ are closely related to those of ordinary analytic functions: there exists a set of functions in class $\mathcal{C}$ which behave like powers of $z$; any function of class $\mathcal{C}$ can be expanded in a uniformly convergent series of these analogs of powers; any function of this class which is regular in a simply-connected domain can be approximated in this domain by the analog of a polynomial (see $\$ 4$ ); singularities of functions of class $C$ have properties analogous to those of analytic functions $[1 \mathrm{c}, \S \S 5-6]$; and so on. Speaking generally, there exists a method of translating properties of analytic functions of a complex variable into properties of complex solutions of the elliptic type equation which belong to class $\mathcal{C}[1 \mathrm{c}, \S 1]$.

Presented to the Society, August 14, 1944; received by the editors July 3, 1944, and, in revised form, October 25, 1944 and April 2, 1945.

1 Numbers in brackets refer to the Bibliography at the end of the paper. 
Before we characterize class $C$ more precisely, it is convenient to introduce the complex notations $z=x+i y, \bar{z}=x-i y, \partial / \partial z$ $=2^{-1}(\partial / \partial x-i \partial / \partial y), \partial / \partial \bar{z}=2^{-1}(\partial / \partial x+i \partial / \partial y)$. Every function of the real variables $x, y$ can be expressed as a function of the conjugate complex variables $z, \bar{z}$. It will be necessary, from time to time, to extend $x$ and $y$ to complex values; $z$ and $\bar{z}$ will then no longer be conjugate, but may then be considered independent complex variables; and our functions will then have been extended to a four-dimensional complex domain. To return to the two-dimensional real domain, it will be sufficient to let $z$ and $\bar{z}$ be complex conjugates. In this notation the Laplace equation assumes the form $U_{z \bar{z}}=0$, and the general elliptic equation assumes the form

$$
\mathrm{L}(u)=U_{z \bar{z}}+a U_{z}+\bar{a} U_{\bar{z}}+c U=0 .
$$

The class $\mathcal{C}$ may now be defined. To every equation (1.1) whose coefficients $a, c$ are entire functions in the real variables $x, y$, their corresponds an entire function $e(z, \bar{z}, t)$ (so-called generating function of the first kind) depending only on the coefficients of $\mathrm{L}$ and such that, if $f$ is an arbitrary analytic function of a complex variable, then

$$
\phi(z, \bar{z})=\int_{t=-1}^{1} e(z, \bar{z}, t) f\left[\frac{z}{2}\left(1-t^{2}\right)\right] d t /\left(1-t^{2}\right)^{1 / 2}
$$

is a complex solution of (1.1) (cf. [1a, \$1]). C consists of the totality of functions $\phi(z, \bar{z})$ defined by (1.2) as $f$ ranges over the field of analytic functions of a complex variable which are regular at the origin.

If $\Phi(z, \bar{z})$ is any real solution of (1.1) which is regular at the origin, then we can determine a complex solution $\phi(z, \bar{z})$ whose real part is $\Phi$ in the following manner. Let $\Phi$ be given as a power series

$$
\Phi(z, \bar{z})=\sum_{m, n=1}^{\infty} D_{m n} z^{m} \bar{z}^{n},
$$

where $D_{m n}=\bar{D}_{n m}$ because $\Phi$ is real, and let $\phi$ be represented by the series

$$
\phi(z, \bar{z})=\sum_{m, n=1}^{\infty} A_{m n} z^{m} \bar{z}^{n}, \quad A_{00} \text { real. }
$$

If we extend $x, y$ to complex values, then it is known that $\phi(z, 0)$ $=2 \Phi(z, 0)-D_{00} \exp \left[-\int_{0}^{z} \bar{a}(0, z) d z\right]$ and $\phi(0, \bar{z})=D_{00} \exp \left[-\int_{0}^{\bar{z}} a(0, \bar{z}) d \bar{z}\right]$ [1c, \$2]. The coefficients $A_{m 0}(m=0,1,2, \cdots)$ and $A_{0 n}(n=1,2, \cdots)$ can be determined from these relations and (1.3); then, because $\phi$ satis- 
fies (1.1), the remaining coefficients $A_{m n}$ can be determined from the $A_{m 0}, A_{0 n}$. Thus, a complex solution $\phi$ may be determined uniquely ${ }^{2}$ from a real solution $\Phi$ in such a way that $\operatorname{Re} \phi=\Phi$; and it can be shown that when $\Phi$ ranges over the totality of real solutions of (1.1) which are regular at the origin then the class of complex solutions $\phi$ obtained by the above procedure is the class $\mathcal{C}[1 \mathrm{c}, \S 3]$. (Note that this is precisely analogous to the manner in which it is possible to determine an analytic function whose real part is a given real harmonic function.)

We may write $\phi(z, \bar{z})=\Phi(z, \bar{z})+i \Psi(z, \bar{z})$, the "conjugate" $\Psi$ being uniquely obtained from the relation $\Psi=-i(\phi-\Phi)$; and it can be shown that $\Psi$ has the same domain of regularity as $\Phi[1 \mathrm{c}]$. Since the complex solution $\phi(z, \bar{z})$ which is obtained from a given real solution $\Phi(z, \bar{z})$ by the above method depends on the choice of origin, the conjugate also depends on the choice of origin. If $\Phi$ is single-valued but has a singular point in its domain, then $\Psi$ will not necessarily be single-valued [1c]. It is evident that $\Psi$ is also a real solution of the differential equation (1.1).

Here again we may note a certain analogy to ordinary analytic functions in that the real and imaginary parts of a complex solution $\phi$ belonging to class $\mathcal{C}$ are connected by a definite relationship, so that if one of them is known the other is uniquely determined. The analogy between class $\mathcal{C}$ and the class of analytic functions of a single complex variable is investigated in more detail in $[1 b, 1 c]$. However, the analogy between these two classes breaks down in an important respect: whereas any rational combination of analytic functions is again an analytic function, the product of two functions of class $C$ in general does not belong to class $\mathcal{C}$. This fact prevents us from applying certain classical methods of the theory of analytic functions when dealing with class $\mathcal{C}$. For instance, it is impossible to develop a method in class $\mathcal{C}$ which is analogous to representing a regular analytic function as the product of functions each of which possesses one simple zero-point (by the use of the Poisson-Jensen formula). It is evidently desirable that such methods should be applicable, however, and in the next section it is shown how to define a class $\mathcal{N}$ of complex solutions of a certain class of nonlinear partial differential equations which is closed under multiplication, and which bears a close relationship to the class $\mathcal{C}$ of complex solutions of elliptic linear equations.

2 That is, to within an imaginary constant multiplied by a certain fixed entire function. 
2. The differential equation $\mathrm{N}(u)=0$ and its real solutions. Let $\mathcal{N}$ be the class of functions whose logarithms belong to $\mathcal{C}$-symbolically, $\log \mathcal{N}=\mathcal{C}$. Because $C$ is closed under addition, $\mathcal{N}$ is closed under multiplication, so that class $\mathcal{N}$ has the property we desire.

Functions of class $\mathcal{N}$ occur in the theory of the two-dimensional heat equation. Let $x, y$ be cartesian coordinates in the plane, and let $U(x, y, t)$ denote the temperature at time $t$ at a point $(x, y)$ in a domain $D$ of the $(x, y)$-plane. If there are no heat sources or sinks in $D$, then $U$ satisfies the equation

$$
h_{\rho} U_{t}=\left(\mu U_{x}\right)_{x}+\left(\mu U_{y}\right)_{y},
$$

where the subscripts denote differentiation with respect to the indicated variable; $h, \rho, \mu$, are respectively the specific heat, the density, and the thermal conductivity.

If we assume that the state is steady and that

$$
\mu=\mathrm{X}(U) Q(x, y),
$$

where $\mathrm{X}$ and $Q$ are nonvanishing, sufficiently many times differentiable and dependent only on $U$ and $x, y$, respectively, then (2.1) becomes

$$
\left[Q(x, y)(\Phi(U))_{x}\right]_{x}+\left[Q(x, y)(\Phi(U))_{y}\right]_{y}=0,
$$

where $\Phi(U)=c_{1} \int_{0}^{U} \mathbf{X}(U) d U+c_{2}, c_{1}, c_{2}$ being constants. If in particular we choose $\Phi(U)=\log U$, that is, $\mathbf{X}(U)=1 / U$, then $U=\exp \Phi$ and the equation (1.7) can be written either in the form

$$
\Phi_{z z}+a \Phi_{z}+a \Phi_{z}=0, \quad \Phi=\log U,
$$

or

$$
\begin{aligned}
\mathrm{N}(U) & \equiv U_{z z}+a U_{z}+\bar{a} U_{z}-U_{z} U_{z} / U=0, \\
a & =4^{-1}\left(Q_{x}+i Q_{y}\right) / Q .
\end{aligned}
$$

We see, therefore, that real parts of functions of class $\mathcal{N}$ represent the temperature distribution under the above described conditions.

REMARK 2.1. If we assume that the law of temperature change is $U(x, y, t)=U^{*}(x, y) e^{-\nu t}$ and $h \rho \nu=C(x, y) / U^{*}$ or $C(x, y) \log U^{*} / U^{*}$, (2.3) becomes

$$
\mathrm{L}(\Phi) \equiv \Phi_{z}+a \Phi_{z}+\vec{a} \Phi_{z}+C / Q=0,
$$$$
\Phi=\log U^{*}
$$

and

$$
\mathrm{L}(\Phi) \equiv \Phi_{z \bar{z}}+a \Phi_{z}+\bar{a} \Phi_{z}+(C / Q) \Phi=0,
$$$$
\Phi=\log U^{*},
$$

respectively. 
It is evident that theorems about functions of class $C$ can, in general, be formulated as theorems about functions of class $\mathcal{N}$. Consequently, a not inconsiderable theory of functions of class $\mathcal{N}$ exists, ready made.

There exist however certain exceptions and we shall in the next section discuss the changes which have to be made in attempting to establish an analogue of the Poisson-Jensen formula.

3. The class $\mathcal{N}$ of complex solutions of $N(u)=0$. As already indicated, the introduction of complex solutions of (2.4) enables us to introduce a corresponding class $\mathcal{N}$ of complex solutions of (2.5). Indeed

$$
\begin{aligned}
w & =\exp \phi=\exp \Phi[\cos \Psi+i \sin \Psi] \\
& =\exp \Phi \cos \Psi+i \exp \Phi \sin \Psi
\end{aligned}
$$

is a solution of (2.5), since $\phi$ is a solution of (2.4). We note that the $|w|=U$ are again (real) solutions of (2.4), and that, corresponding to every solution $U$ of (2.5), there can be found a complex solution $w$ of (2.5) whose absolute value is $U$.

Our approach entails certain complications however. If $\Phi$ is regular in $B$, then $U=\exp \Phi$ will be regular in $B$ and will not vanish there. Since in the following we shall deal with solutions $U$ which are zero or become infinite at some points of $B$, we must consider functions $\Phi$ which have singularities in $B$.

It has already been indicated that in this case the conjugate, and therefore $w=\exp (\Phi+i \Psi)$, are not necessarily single-valued functions. (See [1b, p. 151].)

In order to avoid certain complications we shall assume from now on that the functions $w$ under consideration are regular and do not vanish at the origin. Furthermore, we shall avoid ambiguities in the following way: Every point $z^{(k)}$ in $B$ at which $w$ either vanishes or becomes infinite is connected with the boundary of $B$ by means of a portion of a ray through the origin starting at $z^{(k)}$, directed away from the origin, and ending at the first intersection with the boundary. $w$ is single-valued in the domain $B_{1}$ which is obtained by cutting $B$ along these lines.

Hereafter let $B$ denote a simply connected domain, whose boundary $b$ is a closed curve with a continuous radius of curvature and no double points. Let us assume further that the differential equation has no characteristic values in the domain $B$, so that the only solution $\Phi$ of (2.4) which is regular in $B$ and vanishes on the boundary $b$ of $B$ is $\Phi \equiv 0$.

Definition 3.1. A point, $z^{(0)}$, of $B$ is said to be a zero point (a pole) 
of the first order of $w$ if $w$ vanishes (becomes infinite) at $z^{(0)}$ and if each of the following expressions

$$
h, \log \left|\frac{1}{z-z^{(0)}}\right| \cdot \frac{\partial h}{\partial z}, \log \left|\frac{1}{z-z^{(0)}}\right| \cdot \frac{\partial h}{\partial \bar{z}}
$$

is bounded in the neighborhood of $z=z^{(0)}$. Here $h=\log \left|w /\left(z-z^{(0)}\right)\right|$ in the case of a zero point, and $h=\log \left|w\left(z-z^{(0)}\right)\right|$ in the case of a pole.

Definition 3.2. A solution of the class $\mathcal{N}$,

$$
w=n_{B}\left(z, \bar{z} ; z^{(0)}\right),
$$

of (2.5) is said to be a normalized zero function in the domain $B$, if $n_{B}\left(z, \bar{z} ; z^{(0)}\right)$ has a zero of the first order at $z=z^{(0)}$, and if

$$
\lim _{(\mathbf{z}, \overline{\mathbf{z}}) \rightarrow(b, \bar{b})}\left|n_{B}\left(z, \bar{z} ; z^{(0)}\right)\right|=1 \text {, }
$$

uniformly. $(b, b)$ denotes an (arbitrary) point of the boundary of $B$.

Lemma 3.1. For every point $z^{(0)} \in B$, there exists one and only one normalized zero function $n_{B}\left(z, \bar{z} ; z^{(0)}\right)$.

Proof. Lemma 3.1 is an immediate consequence of the existence of Green's function $\Gamma_{B}\left(z, \bar{z} ; z^{(0)}\right)$ of the equation (2.5) with respect to $B$. See $[2$, p. 8$]$. If $\gamma_{B}\left(z, \bar{z} ; z^{(0)}\right)$ is the corresponding complex solution of (2.5) belonging to class $\mathcal{C}$, then

$$
n_{B}\left(z, \bar{z} ; z^{(0)}\right)=\exp \left[\gamma_{B}\left(z, \bar{z} ; z^{(0)}\right)\right]
$$

will be the required normalized zero function.

REMARK 3.1.

$$
\log \left|n_{B}\left(z, \bar{z} ; z^{(0)}\right)\right|=\Gamma_{B}\left(z, \bar{z} ; z^{(0)}\right) .
$$

Every function $f(z)$ which is meromorphic in ${ }^{3} \mathrm{E}[|z| \leqq 1]$ can be represented there in the form

$$
f(z)=\left[\prod_{k=1}^{\kappa_{1}}\left(\frac{z-\nu_{k}}{1-\nu_{k} z}\right) / \prod_{k=1}^{\kappa_{2}}\left(\frac{z-\mu_{k}}{1-\mu_{k} z}\right)\right] \cdot f_{1}(z)
$$

where $\nu_{k}, \kappa=1,2, \cdots, \kappa_{1}$, and $\mu_{\kappa}, \kappa=1,2, \cdots, \kappa_{2}$, are respectively the zeros and the poles of $w$, and $f_{1}(z)$ is regular and nonvanishing in $\mathrm{E}[|z|<1]$. See $[4$, p. 137].

We can now generalize this result to the case of solutions of (2.5) which belong to the class $\mathcal{N}$.

${ }^{3} \mathrm{E}[\ldots]$ denotes the set of points whose coordinates satisfy the inequalities indicated in brackets. 
THEOREM 3.1. Let $w(z, \bar{z})$ be a (complex) solution of (2.5) belonging to class $\mathcal{N}$ which is defined in the domain $B$ and has continuous boundary values at every point of $b$. Further, assume that w has zero points of first order $^{4}$ at $\nu_{\kappa}, \kappa=1,2, \cdots, \kappa_{1}$, and has poles of first order at $\mu_{k}$, $\kappa=1,2, \cdots, \kappa_{2}$, and that it vanishes at only a finite number of points on the boundary $b$ of $B$.

Then $w$ can be represented in $B_{1}$ in the form

$$
w(z, \bar{z})=\left[\prod_{k=1}^{\kappa_{1}} n_{B}\left(z, \bar{z} ; \nu_{k}\right) / \prod_{k=1}^{\kappa_{3}} n_{B}\left(z, \bar{z} ; \mu_{k}\right)\right] \cdot k(z, \bar{z}),
$$

where $k(z, \bar{z})$ is a solution of (2.5) which belongs to class $\mathcal{N}$, is regular, and does not vanish in $B$, and satisfies

$$
\lim _{(\mathbf{z}, \bar{z}) \rightarrow(b, \bar{b})}|k(z, \bar{z})|=\lim _{(\mathbf{z}, \bar{z}) \rightarrow(b, \bar{b})}|w(z, \bar{z})| .
$$

Proof. The function $\log |w(z, \bar{z})|$ satisfies equation (2.4) and has logarithmic singularities at the points $\nu_{\kappa}$ and $\mu_{\kappa}$. Let $\Gamma_{B}(z, \bar{z} ; \nu)$ denote Green's function of equation (2.4) with respect to the domain $B$. Then

$$
\log |w(z, \bar{z})|-\sum_{k=1}^{\kappa_{1}} \Gamma_{B}\left(z, \bar{z} ; \nu_{k}\right)+\sum_{k=1}^{\kappa_{z}} \Gamma_{B}\left(z, \bar{z} ; \mu_{k}\right)
$$

is a solution of (2.4) which is regular in $B$. Since $\Gamma_{B}(z, \bar{z} ; \nu)$ vanishes on the boundary $b$ and $\log |w(z, \bar{z})|$ is continuous on $b$ except at a finite number of points, expression (3.9) has the same boundary values as $\log |w(z, \bar{z})|$. According to $\$ 2$ there exists a complex solution $\kappa(z, \bar{z})$ of $(2.4)$ in class $\mathcal{C}$ whose real part is (3.9). Writing $k(z, \bar{z})$ $=\exp [\kappa(z, \bar{z})]$, using Lemma 3.1 and Remark 3.1, we obtain (3.8).

It is clear that this procedure enables us to generalize a whole group of results on analytic functions of a complex variable, for instance the theorem of Blaschke, certain theorems on the relation between the growth of functions $w \in \mathcal{N}$ and the density of their zero points and poles in the neighborhood of a point or curve along which they are singular. Since the development of these results does not present any essential difficulty, we shall not carry out these considerations in detail.

REMARK 3.2. Since the existence of Green's function of equation (1.1) is assured by classical results, the problem of generalizing the Poisson-Jensen formula depends on our ability to associate a suitable

4 If the zero is of order $n, n>1$, the corresponding point $\nu_{k}$ will appear $n$ times in the sequence. 
"conjugate" with a given real solution of $L=0$. In order that the resulting complex functions may have valuable properties of their own or in applications, one has to impose certain conditions on the "conjugate," for example, that it is regular in the same domain in which the given function is regular, and so on. A detailed discussion of various types of "conjugate" functions and their properties is given in $[1 \mathrm{~b}, \S 8]$ and $[1 \mathrm{c}, \S \S 2-4]$.

4. A remark concerning the solutions of the general equation (1.7). Using the special form $\Phi=\log U$ we obtained the "canonical representation" (3.8) of functions satisfying this equation of the form (2.5).

(2.5) was rendered linear by setting $\Phi=\log U$ (that is, in (2.2), $\mathbf{X}(U)=1 / U)$. It was then possible to apply the considerations of $\S 3$. In many applications, $\mathbf{X}(U)$ is not of this form. Clearly the method of $\$ 3$ can not now be applied. Many of the properties of the functions in class $\mathcal{C}$ of the linearized equation (1.1) can, however, be applied to the investigation of solutions of (2.3), with $\Phi$ not necessarily equal to $\log U$. In this section we shall consider such an example.

Let $\Lambda$ be the function which is inverse to $\Phi(U)$, that is, such that

$$
U=\Lambda(\Phi),
$$

and assume that $\Lambda$ is an entire function ${ }^{5}$ of its complex argument. For instance, in the case considered in $\S \S 2$ and $3, \Lambda(\Phi) \equiv \exp \Phi$.

Just as in the case of (2.4), where we introduced complex solutions $w$ of (2.5), we now write

$$
u=\Lambda(\phi)
$$

where $\phi$ are solutions of class $C$ of (1.1).

From the fact that in every simply connected domain $B$ which contains the origin and in which $f(z / 2)$ is regular, the solution (1.2) is regular, and its converse, the following result was obtained in the paper $[1 \mathrm{~b}, \mathrm{p} .141]$ : To every equation $\mathrm{L}$ there exists a system of functions

$$
\phi_{n}=2^{-n} z^{n} \int_{-1}^{1} e(z, \bar{z} ; t)\left(1-t^{2}\right)^{n-1 / 2} d t, \quad n=0,1,2, \cdots,
$$

such that every solution $\phi$ of the class $\mathcal{C}$ of $\mathrm{L}(\phi)=0$ which is regular in the circle $x^{2}+y^{2}<\rho^{2}$ can be represented there in the form of

$$
\phi(z, \bar{z})=\sum_{n=0}^{\infty} a_{n} \phi_{n}(z, \bar{z})
$$

- If $\Lambda$ is not an entire function then it is necessary to make obvious changes in the formulation of the statements of this section. 
and can be approximated in every simply connected domain which contains the origin by a "polynomial" $\sum_{n=0}^{m} a_{n}^{(m)} \phi_{n}$. (See also $[1 \mathrm{a}, 82]$.)

We note further that using the classical result of Lindelöf $[3, \mathrm{p}$. 124], we obtain the following conclusion: Let $\phi$ be a solution of $\mathrm{L}(\phi)=0$ and let

$$
\sum_{m=0}^{\infty} \sum_{n=0}^{\infty} A_{m n} z^{m \bar{z}^{n}}
$$

be the power series development of $\phi$ at the origin. Then in every Mittag-Leffler star with the center at the origin in which $\phi$ is regular it may be represented in the form

$$
\lim _{k \rightarrow 0} \sum_{n=0}^{\infty} \frac{C_{n} A_{n 0}}{\Gamma(1+k n)} \phi_{n}, \quad C_{n}=2^{n} \Gamma(n+1) / \pi^{1 / 2} \Gamma(n+1 / 2) .
$$

A fortiori: I. Every solution $u(z, \bar{z})=\Lambda(\phi), \phi \in \mathcal{C}$, of (2.6) can be represented in every circle $x^{2}+y^{2}<\rho^{2}$ in which it is regular in the form

$$
u(z, \bar{z})=\Lambda\left[\sum_{n=0}^{\infty} a_{n} \phi_{n}(z, \bar{z})\right] .
$$

II. In every Mittag-Leffer star with center at the origin in which $u$ is regular it can be represented by

$$
u(z, \bar{z})=\lim _{k \rightarrow 0} \Lambda\left[\sum_{n=0}^{\infty} \frac{C_{n} A_{n 0}}{\Gamma(1+k n)} \phi_{n}(z, \bar{z})\right],
$$

where $A_{n 0}$ are suitably chosen constants. ${ }^{\circ}$

III. Finally in every simply connected domain which includes the origin, and in which $u(z, \bar{z})$ is regular, $u$ can be approximated by

$$
\Lambda\left[\sum_{n=0}^{m} a_{n}^{(m)} \phi_{n}(z, \bar{z})\right] .
$$

REMARK 4.1. If $u(z, \bar{z})$ is given in the form of a power series development $\sum B_{m n} z^{m} \bar{z}^{n}$, then it is possible to express the $A_{n 0}$ in terms of various subsequences of the $B_{m n}$. Substituting the expressions obtained for $A_{n 0}$ into (4.8), we obtain the representation of $u$ in terms of each of these subsequences.

The result III is of interest to applied mathematicians because it leads to a method of determining a solution of (2.3) given by its

- The theorem of Lindelöf mentioned above is a special case of the above statement. We obtain it assuming that $\phi$ is an analytic function of a complex variable and replacing the $C_{n} \phi_{n}$ by the powers $z^{n-1}, n=1,2, \ldots$. 
boundary values. Indeed, for real solutions $U$ of (2.3) we obtain an approximation formula

$$
\Lambda\left[\sum_{n=0}^{m}\left(A_{n}^{(m)} \Phi_{n}(z, \bar{z})+B_{n}^{(m)} \Psi_{n}(z, \bar{z})\right)\right]
$$

analogous to (4.9). Here

$$
\Phi_{n}=\operatorname{Re} \phi_{n}, \quad \Psi_{n}=\operatorname{Im} \phi_{n} .
$$

Suppose now that $U$ is regular in a simply connected domain $B$ and that its values on the boundary $b$ of $B$ are given. We now determine the constants $A_{n}^{(m)}$ and $B_{n}^{(m)}$ in (4.10) in such a way that the expression (4.10) approximates, on $b$, the given boundary values in the "best possible manner." Under appropriate additional conditions it is possible to show that the expressions (4.10) obtained in this manner will converge in $B$ to the required solution when $m \rightarrow \infty$.

\section{Stefan Bergman}

\section{BIBLIOGRAPHY}

a. Zur Theorie der Funktionen, die eine lineare partielle Differentialgleichung befriedigen, Rec. Math. (Mat. Sbornik) N.S. vol. 2 (1937) pp. 1169-1198.

b. Linear operators in the theory of partial differential equations, Trans. Amer. Math. Soc. vol. 53 (1943) pp. 130-155.

c. Certain classes of analytic functions of two real variables and their properties Trans. Amer. Math. Soc. vol. 57 (1945) pp. 299-331.

2. L. LiCHTENSTEIN

Randwertaufgaben der Theorie der linearen partiellen Differentialgleichungen zweiter Ordnung vom elliptischen Typus I, J. Reine Angew. Math. vol. 142 (1913) pp. $1-40$.

3. ERNST LiNDELör

Le calcul des résidus, Paris, 1905.

4. Rolf Nevanlinna

Le thếorème de Picard-Borel et la théorie des fonctions méromorphes, Paris, 1929.

Brown UNIVERSITY 